
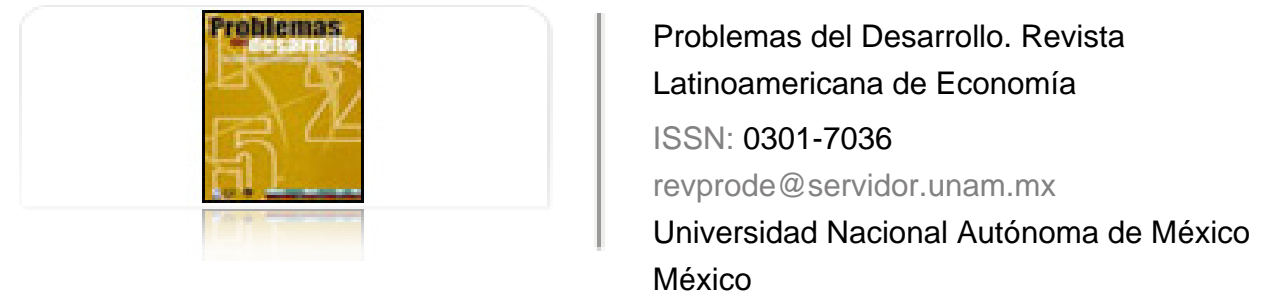

Carrillo-Huerta, Mario M.; Vázquez Mateos, Haydee V.

Desigualdad y polarización en la distribución del ingreso salarial en México

Problemas del Desarrollo. Revista Latinoamericana de Economía, vol. 36, núm. 141, abril-junio, 2005, pp. 109-130

Universidad Nacional Autónoma de México

Distrito Federal, México

Disponible en: http://www.redalyc.org/articulo.oa?id=11820075007

- Cómo citar el artículo

- Número completo

- Más información del artículo

Página de la revista en redalyc.org

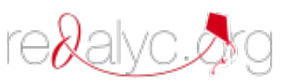

Sistema de Información Científica

Red de Revistas Científicas de América Latina, el Caribe, España y Portugal Proyecto académico sin fines de lucro, desarrollado bajo la iniciativa de acceso abierto 


\section{DESIGUALDAD Y POLARIZACIÓN EN LA DISTRIBUCIÓN DEL INGRESO SALARIAL EN MÉXICO}

\section{Mario M. Carrillo-Huerta* Haydee V.Vázquez Mateos**}

Fecha de recepción: 31 de agosto de 2004. Fecha de aceptación: 17 de mayo de 2005.

\section{Resumen}

Este trabajo muestra los resultados de una investigación acerca de la desigualdad y la polarización de la distribución de los ingresos salariales en México. Se basa en información proveniente de las Encuestas Nacionales de Ingresos y Gastos de los Hogares (ENIGH) realizadas en 1984, 1992, 1994, 1996, 1998, 2000 y 2002, y consiste en calcular indicadores de desigualdad, tales como el coeficiente de Gini, el coeficiente de variación y el índice de Theil, así como indicadores de polarización a partir de la metodología propuesta por Esteban y Ray. Los resultados muestran que si existe desigualdad y polarización en esta distribución, que ambas categorías se están acentuando y que uno de los factores claramente asociados con ellas es la educación, medida como escolaridad.

Palabras clave: desigualdad, polarización social, distribución del ingreso salarial, educación, Encuestas Nacionales de Ingresos y Gastos de los Hogares en México.

\section{Abstract}

This study gives the results of an investigation into the factors affecting inequality and the polarization of wage distribution in Mexico. Based on information from the National Survey of Household Income and Expenditure (ENIGH) in Mexico for 1984, 1992, 1994, 1996, 1998, 2000 and 2002, it consists of calculating inequality indicators such as the Gini coefficient, the variance coefficient and the Theil index, as well as polarization indicators using the methodology proposed by Esteban and Ray. The results show that inequality and polarization in this distribution do indeed exist, both of which are accentuated, and that one of the factors clearly associated with both is education, measured as schooling.

Keywords: inequality, social polarization, wage distribution, education, national survey of household income and expenditure (ENIGH).

* Profesor investigador de El Colegio de Tlaxcala, y profesor del Departamento de Economía de la Universidad de las Américas Puebla. Correo electrónico: mmch@prodigy.net.mx

**Consultora de Asesoría y Consultoría Económica, S.C. Correo electrónico: haydee508@hotmail.com 


\section{Résumé}

Ce travail montre les résultats d'une investigation sur les facteurs qui touchent l'inégalité et la polarisation de la distribution des revenus salariaux au Mexique. Il se base sur l'information des Enquêtes Nationales des Revenus et des Dépenses des Foyers (ENIGH) réalisées au Mexique en 1984, 1992, 1994, 1996, 1998, 2000 et 2002, et il consiste à calculer des indicateurs d'inégalité tels que le coefficient de Gini, le coefficient de variation et l'indice Theil, ainsi que des indicateurs de polarisation à partir de la méthodologie proposée par Esteban et Ray. Les résultats montrent qu'il existe inégalité et polarisation dans cette distribution, que toutes les deux s'accentuent et qu'un des facteurs clairement associé aux deux est l'éducation, mesurée comme scolarité.

Mots clés: inégalité, polarisation sociale, distribution du revenu salarial, education, Enquête Nationale de Revenus et de Dépenses des Foyers (ENIGH).

\section{Resumo}

Este trabalho mostra os resultados duma pesquisa sobre fatores que afeitam a desigualdade e a polarização da distribuição dos ingressos salariais no México. Basea-se na informação que provem das Pesquisas Nacionais de Ingressos e Gastos dos Lares (ENIGH, pelas suas siglas em espanhol) feitas no México em 1984, 1992, 1994, 1996, 1998, 2000 e 2002, e consiste em calcular indicadores de desigualdade tais como o coeficiente de Gini, o coeficiente de variação e o indice de Tehil, além de indicadores de polarização na tal distrubuição, que as duas são acentuadas e que um dos fatores claramente associados com as duas é a educação, medida como escolaridade.

Palavras chave: desigualdade, polarização social, distribuição do ingresso salarial, educação, Pesquisa Nacional de Ingressos y Gastos dos Lares (ENIGH). 


\section{Introducción}

$\mathrm{U}$

no de los fenómenos que en el nivel mundial ha provocado transformaciones importantes en la sociedad ha sido el crecimiento de los asentamientos urbanos. Sin duda, esto se debe a que suele suponerse, en términos generales, que en las ciudades la posibilidad de obtener mayores ingresos y mejor calidad de vida es más elevada que en las zonas rurales.

Por lo regular, las ciudades han sido foco de innovaciones, cultura e invención y han dado lugar a economías de aglomeración, que propician mejores niveles de vida. ${ }^{1}$ Sin embargo, en las urbes también suceden fenómenos adversos que atentan contra la sustentabilidad de esta calidad de vida. Uno de ellos es la desigualdad en los niveles de ingresos de la población en general y de los ingresos salariales, en particular.

En México se observa cierta desigualdad en la distribución del ingreso y algunos autores sugieren que se debe al proceso educativo, pues para obtener de éste beneficios adicionales, es necesario escalar por el sistema. ${ }^{2}$ Es decir, la educación implica costos directos e indirectos, monetarios y no monetarios, que las clases menos privilegiadas difícilmente pueden cubrir. Por esta razón, los pobres sólo se benefician de los niveles educativos más bajos.

El objetivo de este trabajo es caracterizar y medir la desigualdad en la distribución de los ingresos por trabajo del país y examinar algunas de sus posibles causas, incluida la educación. Además, se estudia si dicha distribución no solamente es desigual sino además polarizada, y el efecto que tienen algunas variables como educación, edad, género y tipo de actividad económica, entre otros. La información utilizada proviene de las Encuestas Nacionales de Ingresos y Gastos de los Hogares (ENIGH) que el gobierno mexicano realizó en 1984, 1992, 1994, 1996, 1998, 2000 y 2002 y se consideran los casos de los trabajadores de tiempo completo (entre 35 y 50 horas semanales), que tienen entre 12 y 75 años de edad. ${ }^{3}$

1 En México, durante los últimos 25 años, la población urbana pasó de $45.3 \%$ a $69.5 \%$, a causa de un fenómeno de migración campo ciudad.

2 En los últimos dos decenios, la desigualdad salarial ha aumentado considerablemente en ciertos países desarrollados. Algunos estudiosos apoyan la idea de que se debe a un aumento en la demanda de trabajadores calificados. Por ejemplo, en Estados Unidos la creciente desigualdad salarial ha tenido lugar en, al menos, tres dimensiones: i) aumento considerable en la tasa de rendimiento de la educación superior; ii) un incremento en el rendimiento de los años de experiencia laboral, y iii) incrementos en la desigualdad salarial dentro de grupos de personas definidos por ciertas características observables (Juhn, Murphy y Pierce, 1993).

3 El trabajo incluye información hasta el año 2002, que es el último para el cual existen resultados de la ENIGH, que en México se realiza cada dos años. La última, correspondiente a 2004, no ha sido publicada todavía.

\section{Desaarrollo}


El trabajo consta de cinco secciones. En la segunda se comentan los marcos de referencia de la desigualdad en la distribución del ingreso y de la polarización, así como los indicadores propuestos para medir dichos fenómenos. En la tercera se incluyen algunos antecedentes del caso mexicano y una descripción tanto de la metodología seguida en el estudio, como de las características de los datos y las variables consideradas. En la cuarta sección se presentan los resultados del estudio. Por último, se concluye que la distribución salarial se torna progresivamente más desigual —y, sobre todo, más bipolarizada — durante 19842002, y que uno de los factores que mejor explican este fenómeno es la escolaridad.

\section{Desigualdad y polarización en la distribución del ingreso}

\section{La desigualdad y la polarización}

En una distribución como la del ingreso, la desigualdad mide la dispersión respecto de la media. El valor de ésta ayuda a conocer la eficiencia de la economía para producir el ingreso, mientras que la dispersión indica el grado de equidad con que se distribuye. Por otro lado, el concepto polarización sirve para estudiar en qué medida se conforman diferentes grupos en una distribución. Se analizarán las medidas de desigualdad — los coeficientes de Gini y de variación (cv), y el índice de Theil- y las de polarización — la de Esteban y Ray (1994) y la medida ampliada de Esteban, Gradín y Ray (1999) — que después se calcularán para ser utilizadas en el análisis empírico.

\section{Las medidas de la desigualdad}

Las medidas de desigualdad en la distribución del ingreso pueden ser normativas o positivas (Sen, 1985). Las normativas miden la desigualdad tomando como referencia la noción de bienestar social; las segundas, toman la desigualdad como un hecho y se aplican en un sentido objetivo, que es el caso de los coeficientes de Gini y de variación y del índice de Theil. Una ventaja de estas medidas es que cumplen con los criterios de anonimato, población, ingreso relativo y el de transferencias de Pigou-Dalton. ${ }^{4}$ Por lo tanto, son coherentes con el criterio de Lorenz. ${ }^{5}$

4 El cumplimiento estricto de cuatro propiedades hace del indicador una buena medida de desigualdad: a) satisfacción del principio de transferencia, también conocido como condición Pigou $\sim$ Dalton; $b$ ) in dependencia de la escala de ingreso (el valor de la medida no depende de la unidad monetaria); c) satisfacción del principio de población (la medida deberá ser independiente del tamaño de población analizada); d) posibilidad de descomposición.

5 El principio de transferencias de Pigou Dalton, noción básica que define el concepto desigualdad, establece que si transferimos una unidad monetaria de cualquier individuo hacia otro con un sala rio más bajo, la desigualdad debe disminuir. Estos indicadores muestran sensibilidades distintas a las transferencias que se producen en diferentes polos de la distribución. De este modo, si consideramos una transferencia que reduce la desigualdad y otra que la aumenta, el resultado final dependerá del peso que cada uno asigne a ambas según las posiciones de los individuos afectados en la distribución. 


\section{El coeficiente de Gini}

El coeficiente de Gini, $G$, calcula las diferencias entre todos los pares de individuos y suma todas las diferencias absolutas. Se define como el área que existe entre la curva de Lorenz y la línea de perfecta igualdad o línea de $45^{\circ}$ (Esteban, 1995). Es decir, contabiliza la suma de todas las comparaciones de desigualdades entre dos individuos.

Este índice muestra mayor sensibilidad hacia las transferencias que se producen en el centro de la distribución. Se puede observar que cuando todos los individuos tienen el mismo ingreso: $\mu$, hay perfecta igualdad y el coeficiente de Gini es igual a cero. La máxima desigualdad ocurre cuando una sola persona tiene ingreso $N_{\mu}$ y todos los demás tienen cero; entonces hay $N-1$ diferencias absolutas, cada una de ellas igual a $N_{\mu}$ y el coeficiente de Gini es igual a la unidad. Empíricamente, puede calcularse de la siguiente manera (Sen, 1973):

$$
G(F)=\frac{1}{2 n^{2} \mu} \sum_{i=1}^{n} \sum_{j=1}^{n}\left|y_{i}-y_{j}\right|
$$

donde: $n$ es el total de observaciones; $m$ la media de la distribución del ingreso; $y_{i}, y_{j}$ el ingreso del i-ésimo o j-ésimo individuo.

\section{El indice de Theil}

Una medida de desigualdad, propuesta por Theil (Sen, op. cit.), es la que se desprende de la noción de entropía de la teoría de la información: cuando menos probable sea un suceso, más interesante resulta saber que ha ocurrido. Una fórmula que satisface esa propiedad es el logaritmo del recíproco que establece una función decreciente de $x$.

$$
h(x)=\log \frac{1}{x}
$$

donde: $h(x)$ es el contenido informativo de que el suceso ha ocurrido y $x$, la probabilidad de que cierto acontecimiento suceda. Cuando existen $n$ posibles sucesos $1, \ldots, n$, se toman las probabilidades respectivas, $x_{l}, \ldots, x_{n}$, de tal forma que $x_{1} \geq 0$ y $\sum_{i=1}^{n} x_{1}=1$. La entropía, o el contenido informativo esperado de la situación, es:

$$
H(x)=\sum_{i=1}^{n} x_{1} \log \left(\frac{1}{x_{i}}\right)
$$

donde: $H(x)$ es la medida de entropía esperada de la situación, considerada como la suma del contenido informativo de cada suceso ponderado por la probabilidad respectiva; $x$ es la probabilidad de que $n$ sucesos ocurran. Es evidente que cuanto más se acerquen las $n$ probabilidades $x_{i}$ a $1 / \mathrm{n}$, mayor es la entropía.

\section{Desaarrollo}


Sin embargo, si es la porción del ingreso que recibe el individuo $i$, entonces $H(x)$ es una medida de la igualdad, y si se resta la entropía $H(x)$ de una distribución del ingreso de su valor máximo $\log n$, se obtiene un índice de desigualdad. Ésta es la medida de Theil.

$$
\begin{aligned}
& T=\log n-H(x) \\
& T=\sum_{i=1}^{n} x_{i} \log n x_{i}
\end{aligned}
$$

donde: $x_{i}$ es la porción de ingreso que recibe el individuo $i$, y cuando todas las $x_{i}$ son iguales a $(1 / n), H(x)$ alcanza su valor máximo, $(\log n) ; H(x)$ es la medida de entropía esperada de la situación, considerada como la suma del contenido informativo de cada suceso ponderado por su probabilidad; $T$ es la medida de desigualdad o índice de Theil, y $n$, el número total de datos. ${ }^{6}$

\section{El coeficiente de variación (relativa)}

Se utiliza para expresar la variación como fracción de la media (Montgomery y Ranger, 1996). Si se compara el nivel del ingreso de cada individuo con el ingreso medio (para sumar los valores absolutos de todas las diferencias y considerar entonces la adición como proporción del ingreso total), se obtiene la desviación media relativa, y si antes de sumarlos se elevan al cuadrado, se conseguirá acentuar las diferencias más alejadas de la media. La ecuación del coeficiente de variación es entonces la siguiente:

$$
C V=\frac{s}{\mu}
$$

donde:

$s=\sqrt{\frac{\sum_{i=1}^{n}\left(y_{i}-\mu\right)^{2}}{n-1}}$ es la desviación estándar; $n$ es el tamaño de la población; $y_{i}$ el ingreso del i-ésimo o j-ésimo individuo, y $\mu$ la media de la distribución del ingreso.

Este índice, igual que el de Theil, toma valores iguales o mayores que cero pero no están acotados superiormente. El coeficiente de variación, como los dos anteriores, cum-

6 Esta medida satisface la condición de Pigou Dalton, ya que la transferencia de un individuo rico a otro más pobre reduce el índice de Theil, el cual tiene más sensibilidad a las colas superior e inferior, respectivamente. Toma valores iguales o mayores que cero, sin embargo, no está acotado superior mente. Para In (logaritmo neperiano), el índice de Theil se define como:

$$
T(F)=\frac{1}{n} \sum_{i=1}^{n} \frac{x_{i}}{\mu} \operatorname{In}\left(\frac{x_{i}}{\mu}\right)
$$

donde: $n$ es el tamaño de la población; $y_{i}$ es el ingreso del i $\sim$ ésimo o j ésimo individuo, y $\mu$ la media del ingreso de la distribución del ingreso.

\section{Desarrollo}


ple con la condición de Pigou-Dalton; es decir, la transferencia de un individuo más rico a otro más pobre reduce siempre el valor de Cv (Sen, ibid.).

\section{Las medidas de la polarización}

El concepto polarización trata de explicar un aspecto que la desigualdad no puede: la potencialidad de conflicto existente en una sociedad cuando se forman grupos en torno a alguna característica, por ejemplo, el ingreso. Es decir, los individuos se sienten identificados con otros que pertenecen a su mismo grupo, ya que se encuentran próximos a su nivel de ingreso y ven como antagonistas a quienes están distantes y, por ende, pertenecen a otra clase social. El tamaño de los grupos y la distancia entre ellos pueden causar tensiones, inestabilidad social o la generación de conflicto.

Desde un enfoque estadístico, la polarización social puede interpretarse como el cambio de distribución unimodal a bimodal. En palabras de Sassen (1998), la polarización sucede cuando aumenta el tamaño de las clases alta y baja, al tiempo que la media se reduce; además, la brecha entre la alta y la baja aumenta, es decir, la población se agrupa en torno a polos distantes. ${ }^{7}$

De acuerdo con Esteban y Ray (1994), se estudia la polarización en términos formales "cuando una sociedad se divide en grupos o clases de acuerdo, por ejemplo, al ingreso, de manera que los miembros de una clase tienen ingresos similares, pero las diferentes clases tienen miembros con ingresos muy diferentes" (p. 13).

\section{La medida de Esteban y Ray}

La idea principal detrás de esta medida es definir el antagonismo efectivo en una sociedad determinada por dos tipos de comportamiento: identificación (I) y alienación (A) ${ }^{8} \mathrm{La}$ primera es una actitud respecto de individuos que pertenecen a un mismo grupo. ${ }^{9} \mathrm{La}$ segunda es lo que un individuo siente por otro distante de él en la distribución (dentro del mismo grupo o de otro). ${ }^{10}$ Es claro, entonces, que el antagonismo efectivo es creciente en

$7 \quad$ A pesar de las numerosas medidas de desigualdad que existen, ninguna parece recoger la posición de la desaparición de la clase media. La polarización se refiere a la manera como se mueven las observaciones del centro hacia los lados de manera que, en cualquier distribución con características específicas, se encuentran agrupadas en torno a polos distantes. Esto es importante pues se sabe que en una sociedad en donde la clase media es abundante, se reducen las tensiones sociales, mientras que si es reducida, se agudizan. Teórica y empíricamente, el concepto desigualdad es, por tanto, diferente al de polarización.

8 El antagonismo efectivo entre individuos que pertenecen a diferentes grupos se define en la función continua $T(I, a)$, con $T$ estrictamente creciente en cuanto $(I, a)>0$.

9 La identificación es una función creciente del número de individuos que se encuentran en la subpoblación.

10 Así también, la alienación tiene su función continua. Cuando el ingreso medio de dos individuos es el mismo, es decir, cuando pertenecen al mismo grupo, la alienación es nula.

\section{DeSaarrollo}


relación con la alienación y decreciente con la identificación. La aditividad supone que la polarización es la suma del antagonismo efectivo de todos los individuos de una sociedad. ${ }^{11}$

La medida que se obtiene, conocida como medida de polarización de Esteban y Ray, se expresa como ER en la siguiente ecuación. ${ }^{12}$

$$
E R=A \sum_{i=1}^{k} \sum_{j=1}^{k} p_{i}^{1+\alpha} p_{j}\left|\bar{y}_{i}-\bar{y}_{j}\right|
$$

donde: $A$ es un escalar positivo cuyo objetivo es normalizar; $\alpha$ es el grado de sensibilidad con respecto a la población; $k$ el número de grupos o clases; $p_{i}, p_{j}$ las proporciones de personas en los grupos $i$ y $j$, con respecto al total, y $\overline{y_{i, j}}$ la media del ingreso del grupo $i, j$.

Como puede verse, esta medida es muy sensible a la formación de grupos, así que una misma distribución presentada de dos maneras diferentes arroja resultados muy distintos. Esto es un problema grave, pues el número de grupos en que se divide una sociedad es arbitrario y depende en gran parte de la disponibilidad y presentación de los datos.

La medida que resuelve este problema es la ampliada de Esteban, Gradín y Ray (1999), que se comenta a continuación.

11 De acuerdo con Esteban y Ray (1994) la polarización crece por las siguientes tres razones: a) el grado de heterogeneidad entre los grupos de la distribución; con ello se entiende que el antagonismo efectivo no es decreciente con respecto de la alienación y no es creciente con la identificación. Por ejemplo, una clase extrema se aleja de la central si, y sólo si, la otra clase no se acerca a ella; $b$ ) la homogeneidad interna entre ellos, pues la identificación es función creciente del número de individuos que se encuentran en una misma clase de ingreso. La situación máxima de polarización se alcanza cuando la distribución está fraccionada en dos grandes grupos internamente homogéneos, situados en los extremos de la distribución, cada uno con la mitad de la población; c) los grupos de escaso tamaño son poco relevantes. En este caso, se produce una alteración de las distancias relativas entre los extremos y la clase central, y además el tamaño de los grupos es relevante ya que, por ejemplo, la polarización aumentará ante un acercamiento de la clase pobre a la clase media con respecto de la alta si, y sólo si, la clase baja es más numerosa que la alta. Es decir, la identificación entre los pobres es mayor que la reducción de la alienación hacia los más ricos, sólo si los pobres son más que los ricos. Otro ejemplo muestra que deberá existir una clase media suficientemente grande para que, cuanto más aumente, disminuya la polarización. Las dos últimas características marcan las diferencias con la desigualdad, dado que la mayor homogeneidad interna hace decrecer la primera y crecer la polarización; además, la mayor desigualdad se alcanza cuando un único individuo dispone de todo el ingreso.

12 En ER la variable relevante con la cual se trabaja es el logaritmo natural del ingreso, así que sólo toma valores positivos (no está permitido trabajar con ingreso cero, pues ER no está acotado superior mente). El efecto principal de tomar el logaritmo natural del ingreso es la alteración de las distancias relativas entre ingresos; es decir, dadas dos distancias iguales medidas en dos diferentes tramos de ingreso, la que se toma en un tramo más alto implicará una distancia absoluta mayor (proporcional mente igual) a la que se obtiene en un tramo inferior. En cambio, distancias de ingresos relativos iguales, implican distancias absolutas iguales, independientemente del tramo de ingreso considerado (Gradín, 1994). Los autores escogen esta variable desde la presunción de que sólo las distancias rela tivas entre ingresos son relevantes. A partir de la definición de la medida se puede concluir que la dis tancia entre los ingresos medios, el tamaño de las clases sociales y la sensibilidad respecto de la polarización, conjuntamente determinan la polarización de una distribución. 
La medida ampliada de Esteban, Gradín y Ray

Esteban, Gradín y Ray (1999) proponen una extensión de la medida original, ER, para la distribución dividida en grupos, a la que se le resta una medida del error que se comete cuando se forman los grupos. A diferencia de la ER, esta medida ampliada se utiliza con datos para los cuales ya se ha calculado una función de densidad para la distribución del ingreso (Gradín y Rossi, 2000). Así, si una distribución $F$ está compuesta por $k$ grupos, en la cual la representación de la distribución simplificada de $F$ está dada por una participación $\rho=\left(z_{0}, z_{1}, z_{2}, \ldots, z_{k} ; \overline{y_{1}, \overline{y_{2}}}, \ldots, \overline{y_{k}} ; p_{1}, p_{2}, \ldots, p_{k}\right)$ que delimita $k$ grupos y su i-ésimo grupo está definido por una proporción $p_{i}$ de los trabajadores cuyo salario cae en el intervalo $\left[z_{i-1}, z_{i}\right]$, con salario medio de $\bar{y}_{i}$, cuando se emplea $\rho$ para representar $F$, se incurre en un error de aproximación $\varepsilon(F ; p)$ que se define en términos del grado de dispersión del ingreso de los grupos, medido con el coeficiente de Gini:

$$
\varepsilon\left(F ; p^{c}\right)=G(F)-G\left(p^{c}\right)
$$

donde: $G(F)$ es el coeficiente de Gini de la distribución, y $G\left(\rho^{c}\right)$, el coeficiente de Gini, si suponemos que los grupos al interior son por completo homogéneos.

El error es entonces la diferencia entre la desigualdad — medida por Gini- de la población y la que se tendría si los grupos fueran internamente homogéneos; es decir, si todos los individuos tuvieran la media del ingreso de su grupo. El error representa la falta de identificación interna de los $k$ grupos de la distribución: al substraerlo se toma en cuenta que los grupos no son por completo homogéneos; por tanto, cuanta mayor dispersión interna exista en este grupo, menor será la identificación de sus miembros y, por ende, la polarización. Si se elige la partición óptima $\rho^{*}$, que dado $k$ deja los grupos más identificados o cohesionados internamente, se minimiza el error anterior.

Se tiene entonces la medida ampliada EGR, que arroja la polarización total de la distribución $F$ :

$$
E G R(F ; \alpha, \beta, \rho)=E R(\alpha, \beta)-\beta \varepsilon(F, \rho)
$$

donde: $F$ es la distribución $F ; \beta$ es el peso asignado a la falta de identificación interna; ER representa la medida de polarización de Esteban y Ray (1994) aplicada en $\rho$, (que toma valores entre 0 y 2 cuando está expresada en ingresos relativos a la media, pero no está acotada superiormente cuando el ingreso está expresado en logaritmos. En todo caso, este índice tiene su máximo en 2, pero su mínimo dependerá de $\alpha$ y de la manera de obtener $p$ ). Entonces, EGR tiene su máximo en 2 y su mínimo nunca es menor que $\beta$, pero depende de la manera de obtener $\rho$ y del mismo $\beta$. 
La medida de la bipolarización: cuando $k=2$

La bipolarización se refiere al caso de la formación de dos polos, el cual indica la medida en que la distribución tiende a potenciar sus extremos a costa de la clase media. En este caso especial, la media divide a la población en dos grupos lo más homogéneos posible y luego calcula el grado de bipolarización entre ellos, descontando el grado de falta de identificación interna. La partición óptima divide a la población entre los que están por debajo de la media, una proporción $p_{\mu}=F(\mu)$ y los que están por encima (Gradín y Rossi, 2000). Se expresa como:

$$
P(F, \alpha, \beta)=\left\lfloor p_{1}^{\alpha}+p_{2}^{\alpha}\right\rfloor D(F)-\beta(G(F)-D(F))
$$

donde: $F$ es la distribución $F$; $\alpha$ es el grado de sensibilidad con respecto a la polarización, que varía de 1.0 a $1.6 ; \beta$ es el peso asignado a la falta de identificación interna; $p_{l}, p_{2}$ son las proporciones de individuos en los grupos 1 y 2 con respecto del total; $D(F)=p_{1}-L\left(p_{1}\right)$, es la desviación de la media, y $G$, el coeficiente de Gini de la distribución $F$.

\section{La medida de polarización para el análisis por subpoblaciones}

Puede considerarse cualquier característica de la población, incluidas educación, raza u ocupación, y hacer una partición de la población en $n$ grupos o subpoblaciones. De esta manera, para obtener el nivel de polarización asociado con las características, se define en términos de la medida EGR, la cual mide una polarización de grupo, GP, para las particiones exógenas. Esto probablemente lleve a encontrar valores negativos en la medida EGR, lo cual no es problema si el índice todavía permite ordenar las distribuciones e identificar la intensidad de las diferencias en los niveles de polarización. No obstante, para hacer más sencilla la interpretación de los resultados, se puede normalizar la medida EGR para tener un rango no negativo. ${ }^{13}$ En este caso, la ecuación relevante es la siguiente: ${ }^{14}$

$$
G P\left(F ; \alpha, \beta, p^{c}\right) \equiv P\left(F, \beta, p^{c}\right)-(-\beta)=E R\left(\alpha, p^{c}\right)-\beta\left[\varepsilon\left(F ; p^{c}\right)-1\right]
$$

Esta ecuación modificada se aplica a una distribución agrupada, de acuerdo con una determinada característica del mismo ingreso.

13 El mínimo en el caso de grupos exógenos se obtiene cuando no hay polarización entre los grupos junto con la máxima desigualdad intragrupo; en este caso el mínimo es EGR $=-\beta$.

14 El rango negativo se obtiene restando (-1) a la ecuación (8). 


\section{Estudios empíricos de la desigualdad y su relación con la educación}

Hasta la fecha se ha realizado un buen número de estudios de la desigualdad en la distribución de los ingresos y su relación con la educación. Ahluwalia (1976) encontró una relación negativa importante entre la matrícula escolar y la desigualdad del ingreso en 66 naciones. Psacharopoulos (1987) realizó un estudio de corte transversal con datos de 49 países -incluyendo 37 en vías de desarrollo-, en el cual mostró que la distribución educacional explicaba $29 \%$ del coeficiente de Gini de la desigualdad en el ingreso.

Sin embargo, Zamudio (2000), con datos de las ENIGH de 1984, 1992, 1994 y 1996, muestra que la media de la distribución varía en razón directa la escolaridad, y que ésta reditúa más en los percentiles inferiores y superiores, al reflejar un efecto que reduce la dispersión. ${ }^{15}$ Por otro lado, encuentra que los rendimientos de la escolaridad, medidos en términos de media o mediana condicional, resultaron mayores para los grados educativos altos, lo cual indica que la escolaridad reditúa a los individuos que se encuentran en la parte superior de la distribución y tienen grados educativos más altos. Con esos datos, Lächler (2000) encuentra que la educación incrementa pronunciadamente la desigualdad salarial en México entre 1984 y 1994 y se refleja en una alta dispersión de los salarios y una marcada disminución en los ingresos reales de la gente más pobre.

Como puede verse, los estudios empíricos muestran relaciones, por lo regular, negativas entre la educación y la distribución del ingreso. ${ }^{16}$

\section{Los estudios sobre polarización}

Desde los años ochenta se empezaron a realizar estudios acerca de la posible desaparición de la clase media en Estados Unidos, cuando se suscitó un alarmante descenso de los trabajadores que percibían un ingreso promedio; sin embargo, ninguno de ellos utilizó alguna medida específica para dimensionarla (Gradín, 1994). A partir de 1994, Esteban y Ray (1994), Wolfson (1994), y Esteban, Gradín y Ray (2000) propusieron medidas de polarización.

15 En un modelo de dos grupos, con traslado de trabajadores desde un nivel salarial bajo a uno más alto (y educativo), el grupo puede mostrar un aumento en la varianza del ingreso (o log varianza) hasta que el del nivel educativo alto alcance una proporción total; la proporción precisa depende de la diferencia en los medias y varianzas de los dos grupos.

16 En un estudio para Taiwán, Bourguignon et al. (2000) encuentran que la relación entre la desigualdad de la educación y la del ingreso tiene la forma de una "U" invertida, en la cual un au mento en la desigualdad de la educación, inicialmente aumenta la del ingreso, pero después empieza a bajar dado un cambio en la demanda de destrezas. Winegarden (1991) estima una ecuación en la cual el índice de Gini es la variable dependiente en función de otras, como el promedio de escolaridad, su varianza, el tipo de gobierno y el producto nacional bruto (PNB), y encuentra que un promedio alto en los niveles de escolaridad produce un efecto de equidad en la distribución del ingreso. Sin embargo, la educación y los aumentos en el nivel per capita del ingreso tienen un efecto de desigualdad en los países pobres y de equidad en los más desarrollados.

\section{Desarrrollo}


Gradín (1994) fue el primero que utilizó la medida la polarización de Esteban y Ray para distribuciones discretas, en un estudio de las Comunidades Autónomas de España (CCAA). Para ello, utilizó el logaritmo del ingreso anual medio de los hogares que ofrece la Encuesta de Presupuestos Familiares Anuales 1980-1981. Agrupó los datos de la distribución en tres clases sociales: media, alta y baja, que separó con una línea de pobreza y otra de riqueza. Las seis comunidades con mayor clase media son las de menor polarización y viceversa. Esto da una idea de la relevancia cualitativa del tamaño de la clase media en la polarización.

Esteban, Gradín y Ray (1999) usan la base de datos LIS (Luxemburgo Income Study) para analizar el nivel de polarización, en términos del ingreso familiar, de 1974 a 1997 para cinco países de la OCDE. En el caso de Estados Unidos, tanto la polarización como la desigualdad aumentaron de manera importante, en especial de 1979 a 1986 y de 1991 a 1995. La desigualdad en el Reino Unido sufrió un aumento considerable de 1979 a 1986 y de 1986 a 1991, mientras que la polarización disminuyó en todos los periodos excepto de 1986 a 1991.

Gradín y Rossi (2000) analizan la polarización salarial en Uruguay entre 1986 y 1997 utilizando la extensión de la medida de polarización propuesta por Esteban, Gradín y Ray (1999). La variable objeto de estudio es la remuneración por hora en la ocupación principal del individuo que reporta la Encuesta Continua de Hogares del Instituto Nacional de Estadística de Uruguay. Los resultados muestran que la distribución salarial es progresivamente más desigual y, sobre todo, más bipolarizada.

Gradín (2000) analiza la polarización en España con base en la Encuesta de Presupuestos Familiares utilizando el gasto disponible para 1973-1974, 1980-1981 y 1990-1991. En el primer estudio observa que tanto la desigualdad como la polarización, medida con EGR, disminuyeron significativamente a lo largo del periodo en cuestión. En el siguiente estudio, Gradín explora un nuevo ámbito de la polarización. Primero agrupa con base en el ingreso y el nivel de escolaridad del jefe de familia en los años setenta y la condición socioeconómica para los ochenta. En una segunda etapa, agrupa con base en otras características observables, en lugar de utilizar el ingreso, y concluye que el nivel de escolaridad es la variable más polarizada.

\section{El caso de México}

En esta sección se incluyen tanto los antecedentes del caso de México, como la metodología del estudio. En la primera parte se comentan las modificaciones en la distribución del ingreso en México entre 1950 y 1996, mostrando los cambios más relevantes de acuerdo con el coeficiente de Gini y el criterio de la CEPAL para el mismo periodo. En la segunda parte, se discute la metodología del trabajo. 


\section{Antecedentes de la distribución del ingreso en México: 1950-1996}

De acuerdo con la CEPAL y considerando el valor del coeficiente de Gini, en México se distinguen tres fases de la distribución del ingreso durante el periodo posterior a 1950: a) en la primera fase (1950-1963), la desigualdad en la distribución del ingreso aumentó ligeramente ya que el índice de Gini pasó de 0.515 a $0.546 ; b$ ) en la segunda, de 1963 a 1984, disminuye, pues el índice cayó de 0.546 a $0.472 ; c$ ) en la tercera fase, de 1984 a 1996, el crecimiento de la desigualdad fue intenso, ya que el índice de Gini pasó de 0.472 a 0.610 .

\section{Descripción de la muestra}

La base de datos utilizada para este ejercicio proviene de la Encuesta Nacional de Ingresos y Gastos de los Hogares en México (enIGH) de 1984, 1989, 1992, 1994, 1996, 1998, 2000 y 2002. Cruzando el archivo de las personas entre 12 y 75 años con el de quienes trabajan tiempo completo, se obtuvo la muestra usada en el trabajo, que en forma global incluyó 62933 observaciones. ${ }^{17}$

\section{La metodología del estudio}

Si manejamos los objetivos del trabajo como hipótesis, se tiene lo siguiente: Hipótesis 1: según el coeficiente de Gini (y, comparativamente, el de variación y el índice de Theil), la distribución salarial se torna progresivamente más desigual y, sobre todo, más bipolarizada, y se observa que la educación es una de las causas más importantes del fenómeno. Para esto, se calcula el índice de bipolarización (Gradín y Rossi, 2000).

Para el contraste de esa hipótesis, se calculan las medidas de desigualdad y polarización para analizar cómo se distribuye el ingreso y ver si la distribución es desigual o polarizada; es decir, se calculan las ecuaciones para los coeficientes de Gini, el de variación, y para el índice de Theil. Después se calcula la ecuación (9) para la medida de polarización, la ecuación (10) para la medida de bipolarización, y la ecuación (12), que se muestra a continuación, para calcular la medida EGR por característica de grupo.

17 La muestra corresponde a los diferentes años como sigue: 3088 individuos según la ENIGH 1984; 8182 en 1989; 6728 en 1992; 7915 en 1994; 8804 en 1996; 7036 en 1998; 6 527en 2000, y 14653 de la de 2002. Por otro lado, se consideraron las siguientes características: a) como ingreso principal el del mes anterior a la fecha de la entrevista; $b$ ) se abarcaron sólo los ingresos provenientes del rubro sueldos y salarios; $c$ ) individuos que tuvieran entre 12 y 75 años; $d$ ) se utilizó el ingreso trimestral normalizado (para comparar el ingreso en diferentes años se calculó el ingreso real a precios de septiembre de 1994; para ello se ajustó el ingreso nominal utilizando los índices de precios al consumidor de toda la República); e) se incluyó sólo a los individuos que trabajaran tiempo completo; es decir, entre 35 y 50 horas a la semana, debido a que en muchas oficinas gubernamentales y privadas el tiempo completo consta de sólo siete horas diarias, con dos días de descanso a la sema na, mientras que en otros casos los trabajadores laboran más de ocho horas diarias durante seis y hasta siete días, dependiendo del trabajo que se acumule.

\section{Desarrrollo}




$$
\begin{aligned}
& \square \text { Iny }=\sum_{i=1}^{6} \alpha_{i} X_{i}+\sum_{i=1}^{17} \alpha_{i} \text { Ocp }_{i}+\sum_{i=1}^{7} \alpha_{i} \operatorname{Re}_{i}+\sum_{i=2}^{2} \alpha_{i} \operatorname{Sec}_{i}+\alpha_{i} \text { Trabaj }_{i}+ \\
& +\alpha_{i} \text { Conin }_{i}+\alpha_{i} \text { Mujer }_{i}+\alpha_{i} \text { Sindicato }_{i}+\alpha_{i} \text { Prestación }_{i}+\alpha_{i} \text { Urbano }_{i}+\mu
\end{aligned}
$$

donde: In $Y$, es el logaritmo natural del ingreso salarial real; $X_{i}$ es un vector de variables explicativas numéricas, $\left(X_{1}\right)$ el intercepto, $\left(X_{2}\right)$ educación, $\left(X_{3}\right)$ educación al cuadrado, $\left(X_{4}\right)$ edad, $\left(X_{5}\right)$ edad al cuadrado, $\left(X_{6}\right)$ horas de trabajo semanal. Los otros son vectores de variables dicotómicas que toman el valor de 1 si el individuo pertenece a determinada categoría y 0 en cualquier otro caso: $O c p$, ocupaciones; Reg, región socioeconómica; $S e c$, sector de actividad económica; Trabaj, posición en la ocupación, con la categoría de referencia trabajador sin retribución; ${ }^{18}$ Sindicato indica si el individuo está sindicalizado; Prestación, si recibe prestaciones sociales; Urbano es otra variable dummy que toma el valor de 1 si el individuo pertenece al sector urbano o 0 en caso contrario; Conin señala el tipo de contrato (eventual o permanente); Mujer, el género del individuo.

\section{Los resultados del estudio}

\section{Índices de desigualdad}

Los resultados de los cálculos de los índices de desigualdad se muestran en los cuadros 1 y $1 \mathrm{~A}$, así como en la Gráfica 1. Estos datos muestran que tras un periodo de cierta estabilidad en la distribución del ingreso, se produce una clara tendencia al incremento de la desigualdad en México, sobre todo hasta 1994 y 2002.

Este crecimiento de la desigualdad se reproduce en los tres diferentes índices del periodo considerado: por ejemplo, el índice de Theil crece $82 \%$ frente a $28 \%$ en el caso de Gini, y $14 \%$ en el coeficiente de variación.

\section{Índices de bipolarización}

Aunque la explicación de las causas exactas de la polarización no conciernen a este trabajo, es importante considerar algunos cambios estructurales que influyen en la disminución de la clase media en un país. Ciertamente, uno de los supuestos más importantes es que el elemento que determina el grupo al que pertenece un trabajador es su salario. Pero es posible que sean otras características de los trabajadores las que determinen en realidad dicha pertenencia, tales como tipo de sector, rama, calificación o sexo, entre otras.

18 Trabajador sin retribución se refiere a miembros del hogar que laboraron bajo explotación agropecuaria, de negocio o empresa, propiedad o no del hogar donde residían, siempre y cuando recibieran una ayuda o compensación sin tratarse de un sueldo o pago. Por ejemplo, un aprendiz de peluquero o de albañil. 
Cuadro 1

Medidas de desigualdad en la distribución del ingreso en México, 1984-2002 (valores absolutos)

\begin{tabular}{cccc}
\hline Año & Coeficiente de Gini & Índice de Theil & Coeficiente de variación \\
\hline 1984 & 0.3802500 & 0.2649731 & 0.8710682 \\
1989 & 0.4308097 & 0.3505785 & 1.0738494 \\
1992 & 0.4524739 & 0.3927047 & 1.1519788 \\
1994 & 0.4875782 & 0.4807232 & 1.4325104 \\
1996 & 0.4769700 & 0.4301100 & 1.2050700 \\
1998 & 0.4833038 & 0.4451062 & 1.2530654 \\
2000 & 0.4606354 & 0.4062428 & 1.1868464 \\
2002 & 0.4875960 & 0.4819833 & 0.9972717 \\
\hline
\end{tabular}

Fuente: elaboración propia.

Cuadro 1A

Medidas de desigualdad en la distribución del ingreso en México, 1984-2002 (índices cuando $1984=100$ )

\begin{tabular}{cccc}
\hline Año & Coeficiente de Gini & Índice de Theil & Coeficiente de variación \\
\hline 1984 & 100 & 100 & 100 \\
1989 & 113 & 132 & 123 \\
1992 & 119 & 148 & 132 \\
1994 & 128 & 181 & 164 \\
1996 & 125 & 162 & 138 \\
1998 & 127 & 168 & 144 \\
2000 & 121 & 153 & 136 \\
2002 & 128 & 182 & 114 \\
\hline
\end{tabular}

Fuente: elaboracion propia, con base en datos del Cuadro 1.

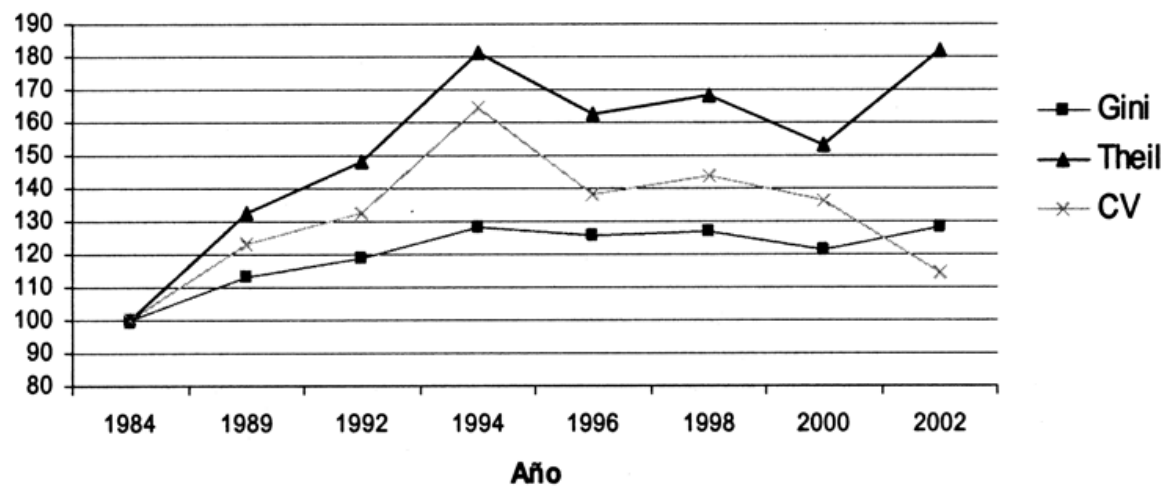

Gráfica 1. Evolución de la desigualdad en México, 1984-2002.

Fuente: elaboración propia, con base en el Cuadro 1A. 
Los datos presentados a continuación consideran dos grupos de ingreso, los cuales se forman a partir de la media: el primero formado por los que se encuentran arriba de la media, y el segundo, por los que se encuentran debajo. Se toman diferentes grados de sensibilidad $(\alpha)$ respecto de la polarización.

En los cuadros 2 y 2A, además de la Gráfica 2, se muestran los resultados del cálculo de la medida de bipolarización EGR. La bipolarización entre los dos grupos salariales se manifiesta en la distancia entre ellos, resultado de la proporción de salarios entre los grupos situados encima y debajo de la media.

Para calcular la polarización mediante el análisis de subpoblaciones se utilizan los datos descritos anteriormente: sólo que no se dividen por su valor promedio general del ingreso, sino por cada característica, como se describe en el Cuadro 3.

Las gráficas 3 y 4 presentan la evolución de la polarización por características, sugiriendo que la mayor polarización salarial se alcanza debido a las diferencias en escolari-

Cuadro 2

Medida de bipolarización ERG $(\beta=0)^{*}$

(valores absolutos)

\begin{tabular}{cccc}
\hline Año & $\alpha=1$ & $\alpha=1.3$ & $\alpha=1.6$ \\
\hline 1984 & 0.25514881 & 0.09179432 & 0.17259346 \\
1989 & 0.27170889 & 0.10000004 & 0.19999994 \\
1992 & 0.28796679 & 0.10989246 & 0.20286805 \\
1994 & 0.29902412 & 0.11543438 & 0.21560210 \\
1996 & 0.30189218 & 0.11890661 & 0.21445335 \\
1998 & 0.31155034 & 0.12459193 & 0.21968151 \\
2000 & 0.29092772 & 0.11294801 & 0.20638915 \\
2002 & 0.31006543 & 0.11532347 & 0.21545572 \\
\hline
\end{tabular}

* Peso asignado a la falta de identificación interna de un grupo.

Fuente: elaboración propia.

Cuadro 2A

Medida de bipolarización ERG $(\beta=0)$ * (índices cuando $1984=100$ )

\begin{tabular}{llll}
\hline Año & \multicolumn{1}{c}{$\alpha=1$} & $\alpha=1.3$ & $\alpha=1.6$ \\
\hline 1984 & 100 & 100 & 100 \\
1989 & 106.4904 & 108.9392 & 104.1505 \\
1992 & 112.8623 & 109.8937 & 105.6441 \\
1994 & 117.1960 & 115.4357 & 112.2754 \\
1996 & 118.3200 & 118.9080 & 111.6771 \\
1998 & 122.1053 & 124.5934 & 114.3997 \\
2000 & 114.0228 & 112.9493 & 107.4777 \\
2002 & 121.5234 & 115.3247 & 112.1985 \\
\hline
\end{tabular}

* Peso asignado a la falta de identificación interna de un grupo.

Fuente: elaboración propia, con base en los datos del Cuadro 2A. 


$$
E R G(\beta=0, \alpha=1.0 ; 1.3 ; 1.6)
$$

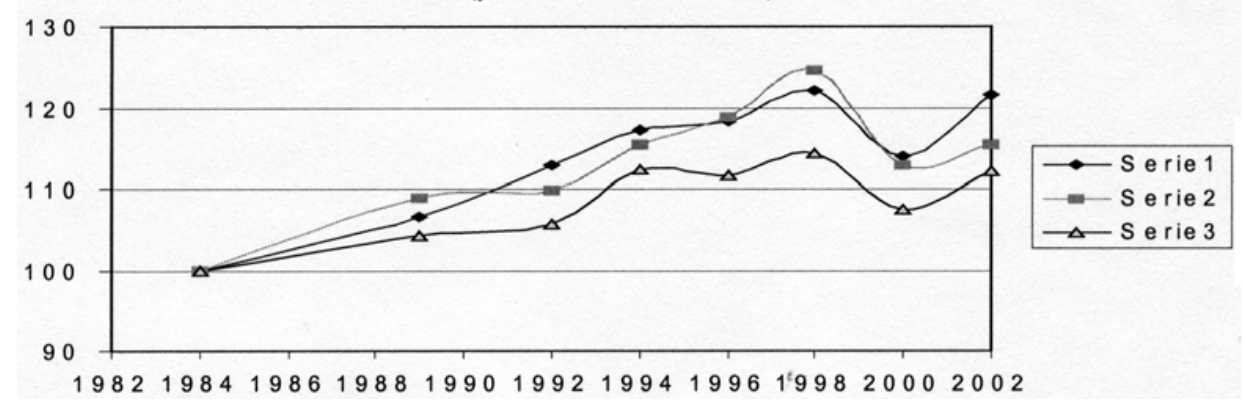

Gráfica 2. Bipolarización en México, 1984-2002.

Fuente: elaboración propia, con base en los datos del cuadro 2A.

dad; asimismo sugieren que la escolaridad muestra los grupos más polarizados entre sí. La ocupación presenta con más claridad una tendencia decreciente, salvo al final del periodo. La edad y rama de la actividad son las características que presentan una senda de polarización creciente en los últimos años. En particular, la edad consigue superar en polarización a la ocupación, y se sitúa tras la escolaridad como la característica que genera más polarización, por lo menos hasta 1998. Las variables de género y horas trabajadas son las que muestran menor polarización, a pesar de que la primera, debido a su carácter dicotómico, presenta una importante polarización entre grupos (aunque es decreciente desde el periodo de inicio).

En otras palabras, se observa que la distribución salarial por grupos se polariza respecto de la educación y la rama de actividad, pero también de la edad de los trabajadores. Aunque los diferenciales de los salarios por horas trabajadas y sexo parecen tener un peso importante, son las que menos contribuyen a este proceso.

\section{Resumen, reflexiones y conclusiones generales}

Resumiendo los resultados obtenidos en el estudio se tiene que:

a) Los coeficientes de Gini, Theil y de variación de la distribución salarial muestran que ésta se torna progresivamente más desigual. El índice de Theil creció 82\% entre 1984 y 2002 frente a $28 \%$ en el caso de Gini y 14\% en el de variación, clara señal de que la desigualdad ha aumentado a partir de 1984.

b) La distribución salarial se torna progresivamente más bipolarizada, medida por el índice EGR, el cual muestra una bipolarización creciente entre los dos grupos salariales, de $28 \%$ entre 1984 y 2002 a 22\%-24\% en 1998.

\section{Desaarrollo}


Cuadro 3

Clasificación de las variables mediante el análisis de subpoblaciones

\begin{tabular}{ll}
\hline Nomenclatura & \multicolumn{1}{c}{ Descripción } \\
\hline Escolaridad (Esc) & Seis niveles de escolaridad: \\
& Primer grupo: $a$ ) escolaridad nula: menos de un año de estudios; $b$ ) escolaridad \\
& escasa: 1 a 5 años; $c$ ) escolaridad baja: 6 años; $d$ ) escolaridad media-baja: 7 a 9 años \\
& Segundo grupo: $e$ ) escolaridad media: 10 a 12 años; $f$ ) escolaridad media-alta: \\
& 13 a 17 años; $g$ ) escolaridad alta, más de 18 años, aquellos que tuvieran algún \\
& posgrado
\end{tabular}

Edad (Edad)

Horas trabajadas $(\mathrm{H})$

Rama (Rama)

Género (Género)

Ocupación (Ocup)
Primer grupo: a) 12 a 16 años; b) 17 a 18 años; c) 19 a 23 años; d) 24 a 29 años Segundo grupo: e) 30 a 39 años; $f$ ) 40 a 49 años; $g$ ) 50 a 59 años; $h$ ) 60 o más

Dos grupos de las horas trabajadas:

a) 35 a 42 horas semanales trabajadas

b) 43 a 50 horas semanales trabajadas

Tres sectores de actividad:

Primer grupo: a) agrícola;

Segundo grupo: $b$ ) industria; $c$ ) servicios

Variables dummy para dos clases de género:

Primer grupo: a) 1 masculino;

Segundo grupo: b) 0 femenino

Dos grupos de ocupaciones:

Primer grupo: a) trabajadores del arte, espectáculos y deportes; supervisores inspectores y otros trabajadores del control en los procesos de producción industrial; y operadores de maquinaria fija de movimiento continuo y equipos en el proceso de producción industrial

Segundo grupo: $b$ ) otros trabajadores con ocupaciones no clasificadas antes

Fuente: elaboración propia.

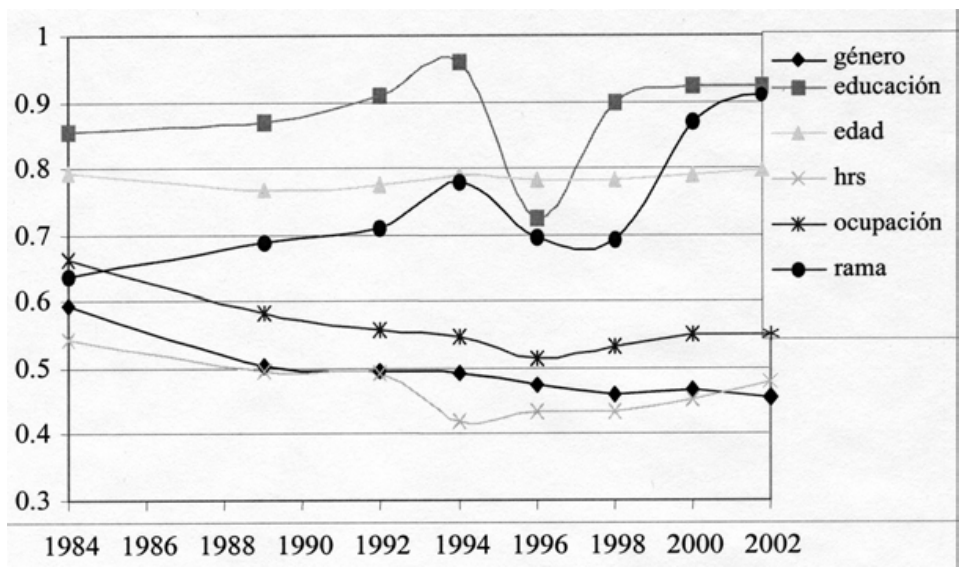

Grafica 3. Polarización de grupos por características: indicador EGR, México, 1984-2002 Fuente: elaboración propia. 


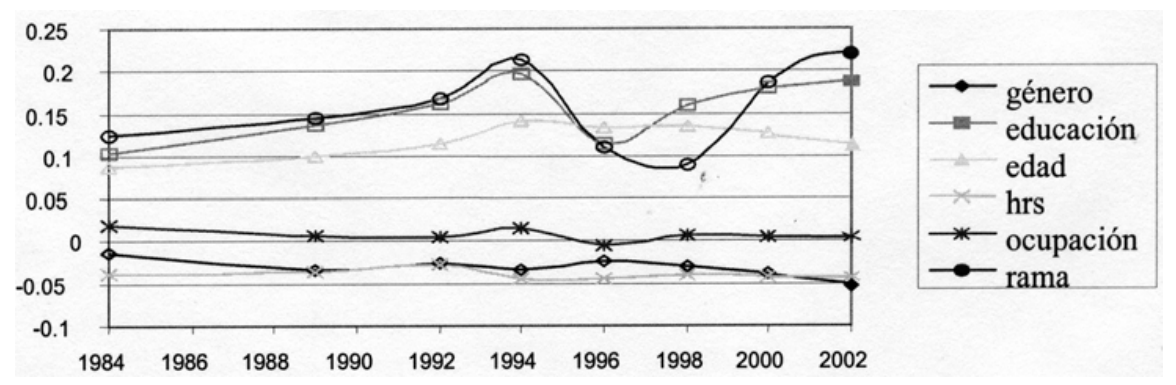

Grafica 4. Polarización de grupos por característica: indicador ER.

Fuente: elaboración propia.

c) El análisis de la evolución de la polarización por características muestra que la escolaridad, que define a los grupos más polarizados entre de 1984 y 2000, ocasiona un incremento de $8 \%$ en dicho periodo. La ocupación presenta una tendencia claramente decreciente, salvo al final del periodo. Edad y rama son las características que indican una senda de polarización bastante creciente en años recientes. En particular, la edad consigue superar en polarización a la ocupación, situándose tras la escolaridad. La categoría del género y las horas trabajadas son las variables que originan menor polarización.

En resumen, puede decirse, entonces, que en México la distribución salarial se torna progresivamente más desigual, y sobre todo más bipolarizada, en el periodo 1984-2002, y que uno de los factores que mejor explican este fenómeno es la escolaridad.

Esos resultados permiten las siguientes relfexiones:

1) En cuanto a la desigualdad, todo parece indicar que tiene relación directa con la recuperación económica: a mayor recuperación, mayor desigualdad. Esto deriva de que entre 1984 y 1994, cuando México inició un periodo de recuperación económica, la desigualdad salarial aumentó. Sin embargo, durante el periodo de dificultades macroeconómicas 1994-2000, hubo una tendencia general a la baja en dicha desigualdad. ${ }^{19}$

2) Lo anterior sugiere que, a diferencia de lo que sucede en países desarrollados, en México el crecimiento económico no afecta uniformemente a los grupos de mayores beneficios, a los más educados y a quienes trabajan en actividades no agrícolas. Esto se corrobora con los resultados del análisis de polarización, que se comentan a continuación.

19 Cabe destacar, sin embargo, que la desigualdad repuntó en los últimos dos años del periodo estudiado (2000 2002), no obstante que la economía no creció. Quizá es muy pronto para detener el curso de la desigualdad, pero es probable que la relación directa se mantenga en el futuro.

\section{Desaarrollo}


Por un lado, aunque la polarización no sigue un patrón igual (por subperiodos) al de la desigualdad, de cualquier manera tiene un crecimiento ascendente en el periodo estudiado. Por otro, el análisis por subgrupos muestra que los grupos beneficiados por el crecimiento económico en México son los más educados y los mayores de edad, mientras que los menores beneficios van a los menos educados y a los más jóvenes.

También cabe destacar que conforme avanza el crecimiento económico, el tipo de actividad contribuye más a la polarización salarial; ello sugiere que quienes realicen labores industriales y de servicios recibirán salarios cada vez mayores que los agropecuarios.

Todo lo anterior permite la siguiente reflexión general: si el proceso de crecimiento económico continúa con las mismas características que hasta ahora, es probable que cause tensiones sociales entre distintos grupos, al hacer mayores las brechas salariales entre la población trabajadora, independientemente de las que pudieran darse por cuestiones políticas.

Este trabajo apunta a las variables en que podría intervenir el Estado a fin de evitar esos problemas. Quizá parezca utópico, pero se antoja que la distribución educativa se haga menos desigual, mediante el apoyo a los grupos de menores niveles; es necesario revisar los ingresos (salarios) en los diferentes sectores.

Bibliografía

Ahluwalia, Montek S., "Income Distribution and Development. Some Stylized Facts", en The American Economic Review, vol. 66, núm. 2, 1976, pp. 128-135.

Becker, Gary S. y Barry R. Chiswick, "Education and the Distribution of Earnings", en The American Economic Review, vol. 56, núm. 1-2, 1966, pp. 358-369.

Blaug, Mark, Economics of Education: A Selected Annotated Bibliography, Oxford, Pergamon Press, 1978.

Blom, Andreas, Nielsen Holm y Verner Lauritz, «Education, Earnings and Inequality in Brazil 1982-1998», Policy Research Working Paper 2686, Washington, The World Bank, 2001.

Bouillon, César, Arianna Legovini y Nora Lustig, Rising Inequality in Mexico: Returns to Household Characteristics and the "Chiapas Effect", Washington, Inter-American Development Bank, 1998.

Bourguignon, Francois, Martin Fournier y Marc Gurgand, Distribution, Development and Education in Taiwan, 1979-1994, París, Universite de París I, 2000.

Bracho, Teresa y Andrés Zamudio, Rendimientos económicos de la escolaridad I: discusión teórica y métodos de estimación, documento de trabajo 30, México, Centro de Investigación y Docencia Económica (CIDE), 1992.

, Rendimientos económicos de la escolaridad II: estimaciones para el caso mexicano, documento de trabajo 31. México, Centro de Investigación y Docencia Económica (CIDE), 1994.

Campbell, M, y B. Stanley, Contemporary Labor Economics, Nueva York, McGraw-Hill, 1995. CEPAL, http://www.cepal.cl/publicaciones/,núm. 64, 1998. —, http://www.cepal.cl/publicaciones/, núm. 78, 2002

Chiswick, Barry, "Earnings, Inequality and Economic Development", en The Quarterly Journal of Economics, vol. 85, núm 1, 1971, pp. 21-39.

- "Income Inequality: Regional Analysis within a Human Capital Framework", en Journal of Economic Literature, vol. 13, núm. 1, 1976, pp. 102-105.

CONAPO, http://www.conapo.gob. $m x / /, 2004$.

Esteban, J. M., Desigualdad y polarización en la distribución interregional de la renta, inédito, Barcelona, Instituto de Análisis Económico, CSIC, 1995.

Esteban, J.M., C. Gradín y D. Ray, Extensions of a Measure of Polarization with an Application to 
the Income Distribution of five OECD Countries, inédito, Nueva York, Syracuse University, 1999.

Esteban, J.M. y D. Ray. "On the Measurement of Polarization”, en Econometrica, vol. 62, núm. 3, 1994, pp. 356-369.

- "Conflict and Distribution", en Journal of Economic Theory, vol. 87, 1999, pp. 379-415.

Fischer, S., R. Dornbusch y R. Schmalensee, Economics, Nueva York, McGraw-Hill, 1998.

Gradín, C., Aproximación a la aplicación de una medida de polarización de rentas, inédito, Barcelona, Universidad Autónoma de Barcelona, 1994.

-, "Polarization by Subpopulations in Spain, 1973-1991", en Review of Income and Wealth, vol. 46, núm. 3, 2000, pp. 276-285.

Gradín, C. y M. Rossi, "Polarización y desigualdad salarial en Uruguay, 1986-1997”, en El Trimestre Económico, vol. LXVII, núm. 2, abril-junio, 2000, pp. 253-276.

Griliches, Zvi, "Estimating the Returns to Schooling: some Econometric Problems", en Econometrica, vol. 45, núm. 1, 1977, pp.1-22.

INEGI, Encuesta Nacional de Ingreso y Gasto de los Hogares, 1984, Muestra Original (ENIGH-89), Aguascalientes, INEGI, 1989, bases de datos y tabulados correspondientes a 1984 y 1989, CD. , Encuesta Nacional de Ingreso y Gasto de los Hogares, 1998. Muestra Original. (ENIGH98), Aguascalientes, INEGI, 1998, bases de datos y tabulados correspondientes a 1992, 1994, 1996 y 1998, CD.

_Encuesta Nacional de Ingreso y Gasto de los Hogares, 2000. Muestra Original (ENIGH-00), Aguascalientes, INEGI, 2000, CD.

Juhn, Chinhui, Kevin M. Murphy y Brooks Pierce, "Wage Inequality and the Rise in Returns to Skill”, en Journal of Political Economy, vol. 101, núm. 3, 1993, pp. 410-442.

Knight, J. B. y R. H. Sabot, "Educational Expansion and the Kuznets Effect"en The American Economic Review, vol. 73, núm. 5, 1983, pp. 1132-1136.

Krueger, Alan B., "How Computers Have Changed the Wage Structure. Evidence from Microdata, 1984-1989", en The Quarterly Journal of Economics, vol. 108, núm. 1, 1993, pp. 33-60.

Kuznetz, Simon, "Economic Growth and Income Inequality", en The American Economic Review, vol. 45, núm. 1, 1955, pp. 1-28.

Lächler, Ulrich, Education and Earnings Inequality in Mexico, Washington, The World Bank, 2000.

Lam, David y Robert F. Schoeni, "Effects of Family Background on Earnings and Returns to Schooling: Evidence from Brazil", en The Journal of Political Economy, vol. 101, núm. 4, 1993, pp. 710-740.
Montgomery, D.C. y G.G. Ranger, Probabilidad y estadística aplicadas a la ingeniería, México, McGraw-Hill, 1996.

Myers, Charles N., Education and National Development in México, Princeton, Princeton University Press, 1965.

Pánuco-Laguette, H. y M. Székely, "La distribución del ingreso y la pobreza en México", en Victor Bulmer Thomas (compilador), El nuevo modelo económico en América Latina, su efecto en la distribución del ingreso y en la pobreza, México, Fondo de Cultura Económica, 1997.

Psacharopoulos, George (compilador), Economics of Education. Research and Studies, Nueva York, Pergamon Press, 1987.

Rivlin, Alice M., "Income Distribution. Can Economics Help?", en The American Economic Review, vol. 67, núm. 2, 1975, pp. 210-235.

Robinson, Sherman, "A Note on the U Hypothesis Relating Income Inequality and Economic Development", en The American Economic Review, vol. 68, núm. 3, 1976, pp. 437-440.

Roll, Eric, Historia de las doctrinas económicas, México, Fondo de Cultura Económica, 1985.

Sassen, A., The Global City: New York, London, Tokyo, Princeton, Princeton University Press, 1998.

Schultz, T.W., Economic Value of Education, Nueva York, Columbia University Press, 1963.

Sen, A., On Economic Inequality, Nueva York, Oxford University Press, 1973.

_ Commodities and Capabilities, Nueva York, Oxford University Press, 1985.

Spense, Michael, "Job Market Signaling", en The Quarterly Journal of Economics, vol. 87, núm. 3, 1973, pp. 355-374.

Taiman, Paul y Terence Wales, "Higher Education, Mental Ability and Screening", en The Journal of Political Economy, vol. 81, núm. 1, 1973, pp. 28-55.

Tinbergen, J., Income Distribution: Analysis and Policies, Amsterdam, North Holland, 1975.

, "Income Distribution: Second Thoughts", en The Economist, vol. 125, núm. 3, 1977, pp. 315-339.

Unikel, L., Gustavo Garza y Adán González, Urbanización y desarrollo económico en México, México, El Colegio de México, 1985.

Velázquez González, Ma. Irene Jovita y José Humberto Angulo Palmero, "Inversión en educación: ¿Un proyecto rentable? El caso de México", tesis de maestría en economía, Puebla, Universidad de las Américas-Puebla, 1998.

Winegarden, C.R., "Schooling and Income Distribution: Evidence from International Data", en Economica, vol. 46, núm 1, 1991, pp. 83-97.

Wise, David A., "Academia Achievement and Job Performance", en The American Economic Review, vol. 67, núm. 3, 1975, pp. 350-366. 
Wolfson, R., "When Inequalities Diverge?", en The American Economic Review, vol. 84, núm. 2, 1994, pp. 353-358.

Wolpin, Kenneth, "Education and Screening", en The American Economic Review, vol. 69, núm. 4, 1977, pp. 949-958.
Yotopoulos, Pan A. y Jeffrey B. Nugent, Economics of Development. Empirical Investigations, Nueva York, Harper \& Row, 1976.

Zamudio, Andrés, La escolaridad y la distribución condicional del ingreso, documento de trabajo, inédito, México, CIDE, 2000. 